\title{
THE LONGITUDINAL KAM-COCYCLE OF A MAGNETIC FLOW
}

\author{
GABRIEL P. PATERNAIN
}

\begin{abstract}
Let $M$ be a closed oriented surface of negative Gaussian curvature and let $\Omega$ be a non-exact 2 -form. Let $\lambda$ be a small positive real number. We show that the longitudinal KAM-cocycle of the magnetic flow given by $\lambda \Omega$ is a coboundary if and only if the Gaussian curvature is constant and $\Omega$ is a constant multiple of the area form.
\end{abstract}

\section{INTRODUCTION}

Let $M$ be a closed oriented surface with negative Gaussian curvature. It is a classical fact that the geodesic flow $\phi$ of $M$ is a contact Anosov flow (cf. 8, 11]). Being contact means that there exists a contact form $\alpha$ on the unit sphere bundle $S M$ such that the vector field $X$ on $S M$ that generates $\phi$ is determined by the equations $\alpha(X) \equiv 1$ and $i_{X} d \alpha \equiv 0$. The Anosov property means that $T(S M)$ splits as $T(S M)=E^{0} \oplus E^{u} \oplus E^{s}$ in such a way that there are constants $C>0$ and $0<\rho<1<\eta$ such that $E^{0}$ is spanned by $X$ and for all $t>0$ we have

$$
\left\|\left.d \phi_{-t}\right|_{E^{u}}\right\| \leq C \eta^{-t} \text { and } \quad\left\|\left.d \phi_{t}\right|_{E^{s}}\right\| \leq C \rho^{t} .
$$

The subbundles are then invariant and Hölder continuous and have smooth integral manifolds, the stable and unstable manifolds, which define a continuous foliation with smooth leaves. The Anosov property immediately implies that $E^{u}$ and $E^{s}$ must be contained in the kernel of the contact form $\alpha$ and thus $\operatorname{Ker} \alpha=E^{u} \oplus E^{s}$ and $E^{u} \oplus E^{s}$ is a $C^{\infty}$ subbundle.

Suppose now that $\lambda$ is a small positive number. Geodesics are smooth curves in $M$ with zero geodesic curvature. Consider instead smooth curves in $M$ with constant geodesic curvature $\lambda$. We will call such curves magnetic geodesics. Through each point $x \in M$, there is a unique magnetic geodesic with velocity $v \in T_{x} M,|v|=1$. Thus magnetic geodesics define also a flow $\phi^{\lambda}$ on $S M$ that we will call the magnetic flow of $M$. For $\lambda$ small, $\phi^{\lambda}$ will still be Anosov, although in general it will not be contact anymore. The flow $\phi^{\lambda}$ is nevertheless still a Hamiltonian flow. A straightforward calculation shows that $\phi^{\lambda}$ is the Hamiltonian flow of the Hamiltonian $H(x, v)=\frac{1}{2}|v|_{x}^{2}$ with respect to the symplectic form on $T M$ given by

$$
-d \alpha+\lambda \pi^{*} \Omega_{a},
$$

where $\Omega_{a}$ is the area form of $M$ and $\pi: T M \rightarrow M$ is the canonical projection. Hence, for small values of $\lambda, \phi^{\lambda}$ is a volume preserving Anosov flow and in fact, it preserves the volume form $\alpha \wedge d \alpha$.

Date: May 2004. 
To any $C^{k}$ volume preserving Anosov flow $\varphi$ on a closed 3-manifold $N$, P. Foulon and B. Hasselblatt [5] associated its longitudinal KAM-cocycle. This is a cocycle that measures the regularity of the subbundle $E^{u} \oplus E^{s}$ and whose definition we now recall. Consider local coordinates (cf. [8, 5]) adapted to the stable and unstable foliations $\psi_{p}:(-\varepsilon, \varepsilon)^{3} \rightarrow N, p \in N$, and denote the coordinate variables $(u, t, s)$. Consider the transversals $\Delta_{p}:=\psi_{p}((-\varepsilon, \varepsilon) \times\{0\} \times(-\varepsilon, \varepsilon))$ and $\Delta_{\varphi_{T}(p)}:=\psi_{\varphi_{T}(p)}((-\varepsilon, \varepsilon) \times\{0\} \times$ $(-\varepsilon, \varepsilon))$. Then $\Delta_{\varphi_{T}(p)} \cap \varphi_{T}\left(\Delta_{p}\right)$ contains local strong unstable and stable manifolds of $\varphi_{T}(p)$, but the two transversals are not in general identical. Let $f_{T}(u, s)$ be the time lengths of the orbit segments between $\Delta_{\varphi_{T}(p)}$ and $\varphi_{T}\left(\Delta_{p}\right)$ and set

$$
K(p, T):=\frac{\partial^{2} f_{T}}{\partial u \partial s}(0,0)
$$

Foulon and Hasselblatt show that $K(p, T)$ is an additive cocycle (i.e. $K(p, T+S)=$ $\left.K\left(\varphi_{T}(p), S\right)+K(p, T)\right)$ and that the cohomology class of $K$ is independent of the adapted coordinates. The main theorem in [5] asserts that $E^{u} \oplus E^{s}$ is always Zygmundregular and that the following are equivalent:

(1) $E^{u} \oplus E^{s}$ is "little Zygmund";

(2) the longitudinal KAM-cocycle is a coboundary;

(3) $E^{u} \oplus E^{s}$ is Lipschitz;

(4) $E^{u} \oplus E^{s}$ is $C^{k-1}$;

(5) $\varphi$ is a suspension or contact flow.

(A continuous function $f: U \rightarrow \mathbb{R}$ on an open set $U \subset \mathbb{R}$ is said to be Zygmundregular $|f(x+h)+f(x-h)-2 f(x)|=O(h)$ for all $x$ in $U$. The function is said to be "little Zygmund" if $|f(x+h)+f(x-h)-2 f(x)|=o(h)$.)

It is well known that for flows, a "choice of time" or equivalently, a choice of speed at which orbits travel gets reflected on the regularity of the corresponding strong stable and strong unstable distributions. The situation is different if we look at the weak unstable and stable bundles $E^{0} \oplus E^{u}$ and $E^{0} \oplus E^{s}$. S. Hurder and A. Katok proved 7] that the weak bundles are always differentiable with Zygmund-regular derivative and there is a cocycle obstruction to higher regularity given by the first nonlinear term in the Moser normal form (this explains why Foulon and Hasselblatt used the terminology "longitudinal KAM-cocycle").

The question we would like to address here is: when is the longitudinal KAMcocycle of the magnetic flow $\phi^{\lambda}$ a coboundary?

Theorem A. Let $K$ be the Gaussian curvature of $M$ and suppose $2 \lambda^{2}+K(x)<0$ for all $x \in M$. Then the longitudinal $K A M$-cocycle of $\phi^{\lambda}, \lambda \neq 0$, is a coboundary if and only if $K$ is constant.

We note that if $\lambda^{2}+K<0$, then $\phi^{\lambda}$ is Anosov (as it can be easily seen from the corresponding Jacobi equation) but there can be other larger values of $\lambda$ for which $\phi^{\lambda}$ is Anosov (cf. [2]). Most likely Theorem A is also true for any value of $\lambda$ for which $\phi^{\lambda}$ is Anosov, but our methods do not yield that much.

There are earlier versions of Theorem A in the literature. Suppose that we replace $\Omega_{a}$ by an arbitrary two-form $\Omega$ and consider as above the Hamiltonian flow 
of $\frac{1}{2}|v|_{x}^{2}$ with respect to the symplectic form given by $-d \alpha+\lambda \pi^{*} \Omega$. We proved in [10] using Aubry-Mather theory (and in any dimensions) that if $\Omega$ is exact, then the corresponding magnetic flow has a $C^{1}$ Anosov splitting only if $\lambda=0$, i.e. the flow is geodesic. Unfortunately, these methods do not carry over to the non-exact case. In an unpublished manuscript [1], J. Boland proved Theorem A under an additional assumption on the metric. He showed that if there is a closed geodesic transverse to which the Gaussian curvature is infinitesimally increasing, then the Anosov splitting is not differentiable for small $\lambda$, unless $\lambda=0$ (how small $\lambda$ had to be was unspecified). His methods were quite different from ours and were based on a probabilistic approach using the Feynman-Kac formula.

Our proof will be based on establishing results for magnetic flows analogous to Theorem 3.6 in [6]. With little extra effort they will yield a more general theorem as we now explain.

Suppose $\Omega$ is an arbitrary non-exact 2 -form and $\phi^{\lambda}$ its associated magnetic flow.

Theorem B. Let $M$ be a closed oriented surface with negative Gaussian curvature. There exists $\lambda_{0}>0$ such that the longitudinal KAM-cocycle of $\phi^{\lambda}$ for $0<|\lambda|<\lambda_{0}$ is a coboundary if and only if $K$ is constant and $\Omega$ is a constant multiple of the area form.

\section{Geometry of $S M$}

Let $M$ be a closed oriented surface, $S M$ the unit sphere bundle and $\pi: S M \rightarrow M$ the canonical projection. The latter is in fact a principal $S^{1}$-fibration and we let $V$ be the infinitesimal generator of the action of $S^{1}$.

Given a unit vector $v \in T_{x} M$, we will denote by $i v$ the unique unit vector orthogonal to $v$ such that $\{v, i v\}$ is an oriented basis of $T_{x} M$. There are two basic 1-forms $\alpha$ and $\beta$ on $S M$ which are defined by the formulas:

$$
\begin{aligned}
& \alpha_{(x, v)}(\xi):=\left\langle d_{(x, v)} \pi(\xi), v\right\rangle \\
& \beta_{(x, v)}(\xi):=\left\langle d_{(x, v)} \pi(\xi), i v\right\rangle .
\end{aligned}
$$

The form $\alpha$ is precisely the contact form that we mentioned in the introduction.

A basic theorem in 2-dimensional Riemannian geometry asserts that there exists a unique 1-form $\psi$ on $S M$ (the connection form) such that

$$
\begin{aligned}
& \psi(V)=1 \\
& d \alpha=\psi \wedge \beta \\
& d \beta=-\psi \wedge \alpha \\
& d \psi=-(K \circ \pi) \alpha \wedge \beta
\end{aligned}
$$

where $K$ is the Gaussian curvature of $M$. In fact, the form $\psi$ is given by

$$
\psi_{(x, v)}(\xi)=\left\langle\frac{D Z}{d t}(0), i v\right\rangle
$$


where $Z:(-\varepsilon, \epsilon) \rightarrow S M$ is any curve with $Z(0)=(x, v)$ and $\dot{Z}(0)=\xi$ and $\frac{D Z}{d t}$ is the covariant derivative of $Z$ along the curve $\pi \circ Z$.

It is easy to check that $\alpha \wedge \beta=\pi^{*} \Omega_{a}$, hence

$$
d \psi=-\pi^{*}\left(K \Omega_{a}\right) .
$$

For later use it is convenient to introduce the vector field $H$ uniquely defined by the conditions $\beta(H)=1$ and $\alpha(H)=\psi(H)=0$. The vector fields $X, H$ and $V$ are dual to $\alpha, \beta$ and $\psi$.

\section{Proof of Theorems A And B}

Let $\Omega$ be an arbitrary smooth 2 -form. We write $\Omega=F \Omega_{a}$, where $F: M \rightarrow \mathbb{R}$ is a smooth function.

Since $H^{2}(M, \mathbb{R})=\mathbb{R}$, there exist a constant $c$ and a smooth 1 -form $\theta$ such that

$$
\Omega=c K \Omega_{a}+d \theta
$$

and $c=0$ if and only if $\Omega$ is exact. Using (11) we have

$$
\omega_{\lambda}:=-d \alpha+\lambda \pi^{*} \Omega=d\left(-\alpha-\lambda c \psi+\lambda \pi^{*} \theta\right) .
$$

The vector field $X_{\lambda}$ that generates $\phi^{\lambda}$ is given by $X_{\lambda}=X+\lambda F V$ since it satisfies the equation $d H=i_{X_{\lambda}} \omega_{\lambda}$. If we evaluate the primitive $-\alpha-\lambda c \psi+\lambda \pi^{*} \theta$ of the symplectic form $\omega_{\lambda}$ on $X_{\lambda}$ we obtain:

$$
\left(-\alpha-\lambda c \psi+\lambda \pi^{*} \theta\right)\left(X_{\lambda}\right)(x, v)=-1-\lambda^{2} F(x) c+\lambda \theta_{x}(v) .
$$

Let us prove the easy part of Theorems A and B. Suppose $M$ has constant curvature and $\Omega$ is a constant multiple of the area form, i.e. $F$ is constant. Then we can choose $\theta=0$ and thus $-\alpha-\lambda c \psi$ is a primitive of $\omega_{\lambda}$ which on the vector field $X_{\lambda}$ is a constant equal to $-1-\lambda^{2} F c$. It follows that $\phi^{\lambda}$ is a contact flow for all values of $\lambda$ except those for which $-1=\lambda^{2} F c$ (in which case the flow is in fact the horocycle flow). If the flow is contact, then of course, its longitudinal KAM-cocyle is a coboundary.

Suppose now that the longitudinal KAM-cocyle is a coboundary. By the main theorem of Foulon and Hasselblatt that we mentioned in the introduction, there is a smooth $\phi^{\lambda}$-invariant 1-form $\tau$ which is equal to 1 on $X_{\lambda}$ and whose kernel is $E^{u} \oplus E^{s}$. By ergodicity, there exists a constant $k$ such that

$$
d \tau=k \omega_{\lambda}
$$

Hence

$$
d\left(\tau+k \alpha+\lambda k c \psi-k \lambda \pi^{*} \theta\right)=0 .
$$

Since $\pi^{*}: H^{1}(M, \mathbb{R}) \rightarrow H^{1}(S M, \mathbb{R})$ is an isomorphism, there exists a closed 1 -form $\delta$ on $M$ and a smooth function $g: S M \rightarrow \mathbb{R}$ such that

$$
\tau+k \alpha+\lambda k c \psi-k \lambda \pi^{*} \theta=\pi^{*} \delta+d g .
$$

Evaluating both sides on $X_{\lambda}$ we obtain

$$
1+k+\lambda^{2} F(x) k c-k \lambda \theta_{x}(v)=\delta_{x}(v)+d g\left(X_{\lambda}\right) .
$$


Since $X$ and $V$ preserve the volume form $\alpha \wedge d \alpha$, then so does $X_{\lambda}=X+\lambda F V$ and thus $\phi^{\lambda}$ preserves the normalized Liouville measure $\mu$ of $S M$. If we integrate (3) with respect to $\mu$ we get

$$
1+k+\lambda^{2} k c \int F d \mu=0
$$

since $\mu$ is invariant under the flip $v \mapsto-v$. It follows that $k$ is a non-zero constant and when $\Omega$ is the area form (Theorem A) we are left with the equation

$$
-k \lambda \theta_{x}(v)-\delta_{x}(v)=d g\left(X_{\lambda}\right)=X_{\lambda}(g) .
$$

Theorem A will now be a consequence of the following result which we prove in the next section.

Theorem 3.1. Let $\Omega$ be the area form and suppose $2 \lambda^{2}+K(x)<0$ for all $x \in M$. If $\omega$ is any smooth 1-form on $M$ such that there is a smooth function $g: S M \rightarrow \mathbb{R}$ for which $\omega_{x}(v)=X_{\lambda}(g)$, then $\omega$ is exact.

If we now apply Theorem 3.1 to the form $-k \lambda \theta-\delta$ we conclude that $\theta$ must be a closed form. But if $\theta$ is closed, $\Omega_{a}=c K \Omega_{a}$ and thus $K$ is constant as desired. This proves Theorem A.

Similarly, Theorem B will be a consequence of the following:

Theorem 3.2. Let $M$ be a closed oriented surface of negative Gaussian curvature and $\Omega$ and arbitrary 2-form. There exists $\lambda_{0}>0$ with the following property. If $G: M \rightarrow \mathbb{R}$ is any smooth function and $\omega$ is any smooth 1-form on $M$ such that there is a smooth function $g: S M \rightarrow \mathbb{R}$ for which $G(x)+\omega_{x}(v)=X_{\lambda}(g)$ for $|\lambda|<\lambda_{0}$, then $G$ is constant and $\omega$ is exact.

If we apply Theorem 3.2 to (3) we conclude as above that $K$ and $F$ must be constant. This proves Theorem B.

\section{Proof of Theorems 3.1 and 3.2}

Theorems 3.1 and 3.2 will be consequences of more general theorems which are the analogue of Theorem 3.6 in [6]. In this section we will try to follow as closely as possible the notation in [6].

Define

$$
\eta^{+}:=(X-i H) / 2
$$

and

$$
\eta^{-}:=(X+i H) / 2 .
$$

Let $L^{2}(S M)$ be the space of square integrable functions with respect to the Liouville measure of $S M$. The next proposition summarizes the main properties of these operators.

Proposition 4.1 ([6]). We have:

(1) $L^{2}(S M)$ decomposes into an orthogonal direct sum of subspaces $\sum H_{n}, n \in \mathbb{Z}$, such that on $H_{n},-i V$ is $n$ times the identity operator; 
(2) $\eta^{+}$extends to a densely defined operator from $H_{n}$ to $H_{n+1}$ for all $n$. Moreover, its transpose is $-\eta_{-}$;

(3) let $A:=\min (-K / 2)$. Then for all $f \in H_{n} \cap$ domain $\eta^{+} \cap$ domain $\eta^{-}$and $n \geq 0$

$$
\left\|\eta^{+} f\right\|^{2} \geq A n\|f\|^{2}+\left\|\eta^{-} f\right\|^{2}
$$

Theorem 4.2. Let $M$ be a closed oriented surface and let $N$ be a non-negative integer. Let $\lambda$ be a real number such that $\lambda^{2} \max \{(N+1), 2\}+K(x)<0$ for all $x \in M$. Let $f: S M \rightarrow \mathbb{R}$ be a smooth function of the form

$$
f=\sum_{|i| \leq N} f_{n}, \quad f_{n} \in H_{n} .
$$

Suppose the integral of $f$ over every periodic orbit of $\phi_{t}^{\lambda}$ is zero. Then there exists a smooth function $g: S M \rightarrow \mathbb{R}$ of the form

$$
g=\sum_{|i| \leq N-1} g_{n}, \quad g_{n} \in H_{n}
$$

such that $X_{\lambda}(g)=f$. (If $N=0$ we interpret this as saying that $g=0$.)

Proof. By the smooth version of the Livsic theorem [9], there exists a smooth function $g$ with $X_{\lambda}(g)=f$. Write

$$
g=\sum_{-\infty}^{\infty} g_{n}
$$

where $g_{n} \in H_{n}$. The equation $X_{\lambda}(g)=f$ is equivalent to the system of equations

$$
\eta^{+} g_{n-1}+\eta^{-} g_{n+1}+i n \lambda g_{n}=f_{n} \quad n=0, \pm 1, \pm 2, \ldots
$$

Since $f_{n}=0$ for $n>N$ we get

$$
\eta^{+} g_{n-1}+\eta^{-} g_{n+1}+i n \lambda g_{n}=0 \quad n>N
$$

Using Proposition 4.1 item 3 and equation (5) we obtain:

$$
\begin{aligned}
\left\|\eta^{+} g_{n+1}\right\|^{2} & \geq\left\|\eta^{-} g_{n+1}\right\|^{2}+A(n+1)\left\|g_{n+1}\right\|^{2} \\
& =\left\|\eta^{+} g_{n-1}+i n \lambda g_{n}\right\|^{2}+A(n+1)\left\|g_{n+1}\right\|^{2} \\
& =\left\|\eta^{+} g_{n-1}\right\|^{2}+2 n \lambda \operatorname{Re}\left\langle\eta^{+} g_{n-1}, i g_{n}\right\rangle+n^{2} \lambda^{2}\left\|g_{n}\right\|^{2}+A(n+1)\left\|g_{n+1}\right\|^{2} .
\end{aligned}
$$

For $n>N+1$ we can also write:

$$
\left\|\eta^{+} g_{n}\right\|^{2} \geq\left\|\eta^{+} g_{n-2}\right\|^{2}+2(n-1) \lambda \operatorname{Re}\left\langle\eta^{+} g_{n-2}, i g_{n-1}\right\rangle+(n-1)^{2} \lambda^{2}\left\|g_{n-1}\right\|^{2}+A n\left\|g_{n}\right\|^{2}
$$

Thus if we set

$$
a_{n}:=\left\|\eta^{+} g_{n}\right\|^{2}+\left\|\eta^{+} g_{n-1}\right\|^{2}
$$

the last two inequalities imply for $n>N+1$ :

$$
\begin{aligned}
a_{n+1} & \geq a_{n-1}+2 n \lambda \operatorname{Re}\left\langle\eta^{+} g_{n-1}, i g_{n}\right\rangle+2(n-1) \lambda \operatorname{Re}\left\langle\eta^{+} g_{n-2}, i g_{n-1}\right\rangle \\
& +n^{2} \lambda^{2}\left\|g_{n}\right\|^{2}+A(n+1)\left\|g_{n+1}\right\|^{2}+(n-1)^{2} \lambda^{2}\left\|g_{n-1}\right\|^{2}+A n\left\|g_{n}\right\|^{2}
\end{aligned}
$$


Now we compute using equation (15) again:

$$
\begin{aligned}
\operatorname{Re}\left\langle\eta^{+} g_{n-1}, i g_{n}\right\rangle & =\operatorname{Re}\left\langle g_{n-1},-\eta^{-}\left(i g_{n}\right)\right\rangle \\
& =\operatorname{Re}\left\langle g_{n-1},-i\left(-i(n-1) \lambda g_{n-1}-\eta^{+} g_{n-2}\right)\right\rangle \\
& =-(n-1) \lambda\left\|g_{n-1}\right\|^{2}-\operatorname{Re}\left\langle\eta^{+} g_{n-2}, i g_{n-1}\right\rangle
\end{aligned}
$$

for $n>N+1$. Hence

$$
\begin{array}{r}
2 n \lambda \operatorname{Re}\left\langle\eta^{+} g_{n-1}, i g_{n}\right\rangle+2(n-1) \lambda \operatorname{Re}\left\langle\eta^{+} g_{n-2}, i g_{n-1}\right\rangle \\
=-2 n(n-1) \lambda^{2}\left\|g_{n-1}\right\|^{2}-2 \lambda \operatorname{Re}\left\langle\eta^{+} g_{n-2}, i g_{n-1}\right\rangle .
\end{array}
$$

This yields for $n>N+1$ :

$$
\begin{aligned}
a_{n+1} & \geq a_{n-1}-2 \lambda \operatorname{Re}\left\langle\eta^{+} g_{n-2}, i g_{n-1}\right\rangle \\
& +n^{2} \lambda^{2}\left\|g_{n}\right\|^{2}+A(n+1)\left\|g_{n+1}\right\|^{2}-(n-1)(n+1) \lambda^{2}\left\|g_{n-1}\right\|^{2}+A n\left\|g_{n}\right\|^{2} .
\end{aligned}
$$

For $n>N+2$ we can also write:

$$
\begin{aligned}
a_{n} & \geq a_{n-2}-2 \lambda \operatorname{Re}\left\langle\eta^{+} g_{n-3}, i g_{n-2}\right\rangle \\
& +(n-1)^{2} \lambda^{2}\left\|g_{n-1}\right\|^{2}+A n\left\|g_{n}\right\|^{2}-(n-2) n \lambda^{2}\left\|g_{n-2}\right\|^{2}+A(n-1)\left\|g_{n-1}\right\|^{2} .
\end{aligned}
$$

Thus if we set

$$
b_{n}:=a_{n}+a_{n-1}
$$

the last two inequalities imply for $n>N+2$ :

$$
\begin{aligned}
b_{n+1} \geq & b_{n-1}+2(n-2) \lambda^{2}\left\|g_{n-2}\right\|^{2}-(n-2) n \lambda^{2}\left\|g_{n-2}\right\|^{2} \\
& +(n-1)\left(A-2 \lambda^{2}\right)\left\|g_{n-1}\right\|^{2}+\left(2 A n+\lambda^{2} n^{2}\right)\left\|g_{n}\right\|^{2}+A(n+1)\left\|g_{n+1}\right\|^{2} .
\end{aligned}
$$

This is the basic recurrence inequality that we will use to prove the theorem. Let us call

$$
\begin{aligned}
r_{n}:=-(n-2)^{2} \lambda^{2}\left\|g_{n-2}\right\|^{2}+ & (n-1)\left(A-2 \lambda^{2}\right)\left\|g_{n-1}\right\|^{2}+\left(2 A n+\lambda^{2} n^{2}\right)\left\|g_{n}\right\|^{2} \\
& +A(n+1)\left\|g_{n+1}\right\|^{2}
\end{aligned}
$$

which is defined for $n>N+2$. We have:

$$
b_{n+1} \geq b_{n-1}+r_{n} .
$$

Consider now an positive integer $n$ of the form $n=N+3+2 k$ for $k \geq 0$ and let $j$ be another integer with $k \geq j \geq 0$. Using (6) we obtain:

$$
b_{n+1} \geq b_{N+2+2 j}+r_{n}+r_{n-2}+\cdots+r_{N+3+2 j} .
$$

Note that

$$
\begin{gathered}
r_{n}+r_{n-2}=-(n-4)^{2} \lambda^{2}\left\|g_{n-4}\right\|^{2}+(n-3)\left(A-2 \lambda^{2}\right)\left\|g_{n-3}\right\|^{2}+2 A(n-2)\left\|g_{n-2}\right\|^{2} \\
+2(n-1)\left(A-\lambda^{2}\right)\left\|g_{n-1}\right\|^{2}+\left(2 A n+\lambda^{2} n^{2}\right)\left\|g_{n}\right\|^{2}+A(n+1)\left\|g_{n+1}\right\|^{2} .
\end{gathered}
$$

If we now suppose that $A-\lambda^{2} \geq 0$, then a simple calculation gives:

$$
\begin{gathered}
r_{n}+r_{n-2}+\cdots+r_{N+3+2 j} \geq-(N+1+2 j)^{2} \lambda^{2}\left\|g_{N+1+2 j}\right\|^{2} \\
+(N+2+2 j)\left(A-2 \lambda^{2}\right)\left\|g_{N+2+2 j}\right\|^{2} .
\end{gathered}
$$


Using (71) we obtain:

$$
\begin{gathered}
b_{n+1} \geq\left\|\eta^{+} g_{N+2+2 j}\right\|^{2}+2\left\|\eta^{+} g_{N+1+2 j}\right\|^{2}+\left\|\eta^{+} g_{N+2 j}\right\|^{2} \\
-(N+1+2 j)^{2} \lambda^{2}\left\|g_{N+1+2 j}\right\|^{2}+(N+2+2 j)\left(A-2 \lambda^{2}\right)\left\|g_{N+2+2 j}\right\|^{2},
\end{gathered}
$$

which combined with Proposition 4.1 item 3 gives:

$$
b_{n+1} \geq 2(N+2+2 j)\left(A-\lambda^{2}\right)\left\|g_{N+2+2 j}\right\|^{2}
$$

$$
+(N+1+2 j)\left(2 A-(N+1+2 j) \lambda^{2}\right)\left\|g_{N+1+2 j}\right\|^{2}+A(N+2 j)\left\|g_{N+2 j}\right\|^{2} .
$$

Analogously, if we take a positive integer $n$ of the form $n=N+3+2 k+1$ for $k \geq 0$ and let $j$ be another integer with $k \geq j \geq 0$ we obtain:

$$
b_{n+1} \geq 2(N+3+2 j)\left(A-\lambda^{2}\right)\left\|g_{N+3+2 j}\right\|^{2}
$$

$$
+(N+2+2 j)\left(2 A-(N+2+2 j) \lambda^{2}\right)\left\|g_{N+2+2 j}\right\|^{2}+A(N+1+2 j)\left\|g_{N+1+2 j}\right\|^{2} \text {. }
$$

Suppose now that $A-\lambda^{2}>0$ and $2 A-(N+1) \lambda^{2}>0$. Since $g$ is a smooth function, the functions $g_{n}$ must tend to zero in the $L_{2}$-topology as $n$ tends to infinity. Thus $b_{n} \rightarrow 0$, which can only occur if $g_{N}=g_{N+1}=g_{N+2}=0$ in view of (8) for $j=0$. If we now use that $g_{N+1}=g_{N+2}=0$ in (9) for $j=0$, the fact that $b_{n} \rightarrow 0$ shows that $g_{N+3}=0$. If we continue in this fashion using (8) and (9) for all values of $j$ we get that $g_{n}=0$ for all $n \geq N$.

Analogously one can prove that $g_{n}=0$ for $n \leq-N$.

Theorem 3.1 follows right away from Theorem 4.2 given a 1-form $\omega$ on $M$, regarded as a function $\omega: T M \rightarrow \mathbb{R}$, we can consider its restriction to $S M$ which lies in $H_{1} \oplus H_{-1}$. (If we write $\omega=\omega_{1}+\omega_{-1}$, then $\bar{\omega}_{1}=\omega_{-1}$.) Theorem 4.2 tell us that there exists $g \in H_{0}$ such that $\omega=X_{\lambda}(g)$. But if $g \in H_{0}$, then it is the pull back of a smooth function $h: M \rightarrow \mathbb{R}$ and thus $X_{\lambda}(g)=d h$ and $\omega$ is exact as desired.

Similarly, Theorem 3.2 follows immediately from the following theorem. Its proof is quite similar to that of Theorem 4.2, so we will only indicate the main steps.

Theorem 4.3. Let $M$ be a closed oriented surface of negative Gaussian curvature and let $\Omega$ be an arbitrary smooth 2-form. Let $N$ be a non-negative integer. There exists a positive number $\lambda_{0}$ which depends on the metric, $\Omega$ and $N$ such that for all $\lambda \in\left[0, \lambda_{0}\right)$ the following property holds: let $f: S M \rightarrow \mathbb{R}$ be a smooth function of the form

$$
f=\sum_{|i| \leq N} f_{n}, \quad f_{n} \in H_{n}
$$

Suppose the integral of $f$ over every periodic orbit of $\phi_{t}^{\lambda}$ is zero. Then there exists a smooth function $g: S M \rightarrow \mathbb{R}$ of the form

$$
g=\sum_{|i| \leq N-1} g_{n}, \quad g_{n} \in H_{n}
$$


such that $X_{\lambda}(g)=f$. (If $N=0$ we interpret this as saying that $g=0$.)

Proof. We will suppose without loss of generality that the metric is normalized so that $K \leq-2$.

Using

$$
\eta^{+} g_{n-1}+\eta^{-} g_{n+1}+i n \lambda F g_{n}=0, \quad n>N,
$$

the same proof of Theorem 4.2 shows that (with the same definition of $b_{n}$ ) for $n>$ $N+2$ we have:

$$
\begin{aligned}
b_{n+1} \geq & b_{n-1}-2(n-1)\left\|\lambda F g_{n-1}\right\|^{2}-(n-2)^{2}\left\|\lambda F g_{n-2}\right\|^{2}+n^{2}\left\|\lambda F g_{n}\right\|^{2} \\
& +(n-1) A\left\|g_{n-1}\right\|^{2}+2 A n\left\|g_{n}\right\|^{2}+A(n+1)\left\|g_{n+1}\right\|^{2} \\
& +2(n-2) \operatorname{Re}\left\langle g_{n-2},-i \eta^{-}(\lambda F) g_{n-1}\right\rangle+2 n \operatorname{Re}\left\langle g_{n-1},-i \eta^{-}(\lambda F) g_{n}\right\rangle .
\end{aligned}
$$

We now observe:

$$
\begin{gathered}
2(n-2) \operatorname{Re}\left\langle g_{n-2},-i \eta^{-}(\lambda F) g_{n-1}\right\rangle+2 n \operatorname{Re}\left\langle g_{n-1},-i \eta^{-}(\lambda F) g_{n}\right\rangle \\
\geq-(n-2)\left\|g_{n-2}\right\|^{2}-2(n-1)\left\|\eta^{-}(\lambda F) g_{n-1}\right\|^{2}-n\left\|g_{n}\right\|^{2} .
\end{gathered}
$$

Since $M$ is compact there exists a positive constant $c$ such that for all $n$ and $g$ we have

and thus

$$
\begin{gathered}
\left\|\eta^{-}(F) g_{n}\right\|^{2} \leq c\left\|g_{n}\right\|^{2} \\
\left\|F g_{n}\right\|^{2} \leq c\left\|g_{n}\right\|^{2}
\end{gathered}
$$

$$
\begin{aligned}
b_{n+1} \geq & b_{n-1}-(n-2)^{2}\left\|\lambda F g_{n-2}\right\|^{2}+n^{2}\left\|\lambda F g_{n}\right\|^{2}-(n-2)\left\|g_{n-2}\right\|^{2} \\
& +(n-1)\left(A-4 c \lambda^{2}\right)\left\|g_{n-1}\right\|^{2}+(2 A-1) n\left\|g_{n}\right\|^{2}+A(n+1)\left\|g_{n+1}\right\|^{2} .
\end{aligned}
$$

By choosing $\lambda$ small enough we may suppose that $A-4 c \lambda^{2} \geq 0$. Our normalization of the metric says that $A \geq 1$ and hence

$$
b_{n+1} \geq b_{n-1}-(n-2)^{2}\left\|\lambda F g_{n-2}\right\|^{2}+n^{2}\left\|\lambda F g_{n}\right\|^{2}-(n-2)\left\|g_{n-2}\right\|^{2}+n\left\|g_{n}\right\|^{2} .
$$

This implies

$$
b_{n+1} \geq b_{N+2}-(N+1)^{2}\left\|\lambda F g_{N+1}\right\|^{2}-(N+1)\left\|g_{N+1}\right\|^{2} .
$$

Using the definition of $b_{n}$ and Proposition 4.1 item 3 we obtain:

$$
b_{n+1} \geq A(N+2)\left\|g_{N+2}\right\|^{2}+\left[(2 A-1)(N+1)-\lambda^{2} c(N+1)^{2}\right]\left\|g_{N+1}\right\|^{2}+A N\left\|g_{N}\right\|^{2}
$$

and if we now choose $\lambda$ small enough so that

$$
(2 A-1)(N+1)-\lambda^{2} c(N+1)^{2}>0
$$

then the same argument as in Theorem 4.2 shows that $g_{N}=g_{N+1}=g_{N+2}=0$. Now it follows easily that $g_{n}=0$ for all $n \geq N$. 
Remark 4.4. It is quite likely that one can prove (and perhaps improve) Theorems 4.2 and 4.3 by proving first a Pestov's identity for magnetic flows and then proceeding as in [3, 4].

\section{REFERENCES}

[1] J. Boland, On the regularity of the Anosov splitting and conjugacy types for magnetic field flows, preprint 1999.

[2] K. Burns, G.P. Paternain, Anosov magnetic flows, critical values and topological entropy, Nonlinearity 15 (2002) 281-314.

[3] C.B. Croke, V.A. Sharafutdinov, Spectral rigidity of a negatively curved manifold, Topology $\mathbf{3 7}$ (1998) 1265-1273.

[4] N.S. Dairbekov, V.A. Sharafutdinov, Some problems of integral geometry on Anosov manifolds, Ergod. Th. and Dynam. Sys. 23 (2003) 59-74.

[5] P. Foulon, B. Hasselblatt, Zygmund strong foliations, Israel J. Math. 138 (2003) 157-169.

[6] V. Guillemin, D. Kazhdan, Some inverse spectral results for negatively curved 2-manifolds, Topology 19 (1980) 301-312.

[7] S. Hurder, A. Katok, Differentiability, rigidity and Godbillon-Vey classes for Anosov flows, Publ. Math. IHES 72 (1990) 5-61.

[8] A. Katok, B. Hasselblatt, Introduction to the modern theory of dynamical systems, Encyclopedia of Mathematics and its Applications 54, Cambridge University Press, 1995.

[9] R. de la Llave, J.M. Marco, R. Moriyon, Canonical perturbation theory of Anosov systems and regularity for the Livsic cohomology equation, Ann. Math. 123 (1986) 537-611.

[10] G.P. Paternain, On the regularity of the Anosov splitting for twisted geodesic flows, Math. Res. Lett. 4 (1997) 871-888.

[11] G.P. Paternain, Geodesic flows, Progress in Mathematics, 180 Birkäuser 1999.

Department of Pure Mathematics and Mathematical Statistics, University of Cambridge, Cambridge CB3 0WB, England

E-mail address: g.p.paternain@dpmms.cam.ac.uk 